Ittishal Educational Research Journal

July 2020, Vol. 1, No. 01, p. 72 - 81

http://journal.ittishal.net/index.php/ierj

DOI: 10.51425/ierj.v1i1.4

\title{
Methodological Problems of Teaching Arabic to Non-Native Speakers in Indonesia
}

\author{
M. Ilham Muchtar \\ Muhammadiyah University of Makassar, Indonesia \\ ilhammuchtar@unismuh.ac.id \\ Jamal Fauzi \\ Faculty of Dar Al-Ulum, Cairo, Egypt \\ gamal_fawzi@darelom.cu.edu.eg
}

\begin{abstract}
The Arabic language is a decent place in Indonesian society because it is a religious language that coincided with the entry of Islam in this country. The growing interest in it through the ages and continued to try to raise its status continuously until the field of education witnessed a remarkable development in the last decade. However, this status does not necessarily mean that this language has received what it deserves as a religious and foreign language from the engineering and policy of advanced education. The current status of Arabic language education in Indonesia is a transitional phase, as some promising developments are beginning to take place on the one hand, and it is still facing a number of thorny problems on the other.

The teaching of Arabic in most institutes in Indonesia is done in a way that explains the rules and the translation of texts into the Indonesian language. In addition, the evaluation system used to measure the learner's linguistic achievement is largely focused on linguistic aspects.

The teaching of Arabic language on such a method is a traditional trend and it has to be changed and developed, and since some of the significant shortcomings related to the curriculum, it requires searching for the places of this deficiency in the curriculum of teaching Arabic in the institutes. The framework of the study is determined in the problems of the curriculum of the Arabic language in terms of objectives, content, method and means of education and evaluation. The study took its place in the language institutes of the Asia Muslims Charity Foundation (AMCF) in Indonesia.
\end{abstract}

Keywords: methodological problems; transitional; linguistic performance 


\section{المشكلات المنهجية في تعليم اللغة العربية للناطقين بغيرها في إندونيسيا \\ (تجربة في المعاهد اللغوية التابعة لمؤسسة مسلمي أَسيا الخيرية أنموذجاً)}

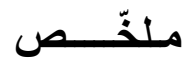

لقد كانت للغة العربية لأبناء الإندونيسين شأنا عظيما ويرجع هذا الفضل لأن اللغة العربية متز امنة مع بداية بزوغ الإسلام

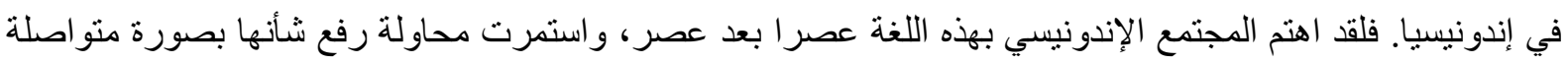

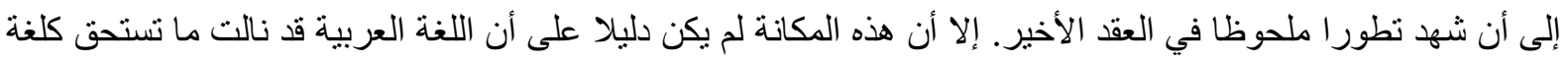

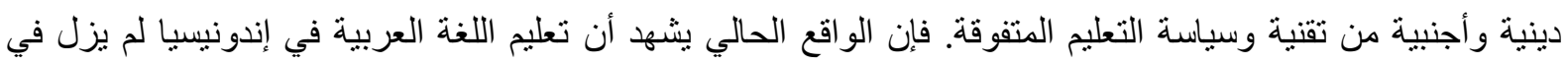

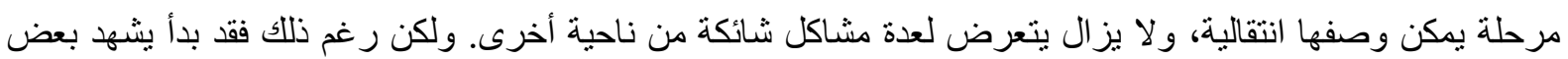
التطور ات الملحوظة في مجال تعليمها من ناحية.

إن تعليم اللغة العربية في معظم المعاهد في إندونيسيا يتم بطريقة شرح القواعد وترجمة النصوص إلى اللغة الإندونيسية،

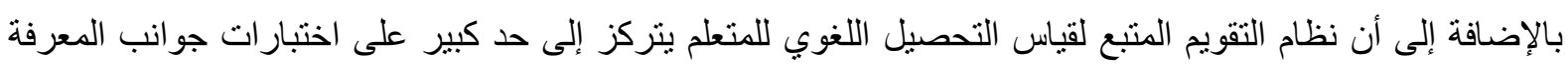
اللغوية وقد لا يهتم بجانب الأداء اللغوي.

تعليم اللغة العربية على مثل هذا الأسلوب هو تقليدي الإتجاه فيضطر إلى تغييره وتطويره، وبما أن بعض مظاهر القصور الملحوظ يتعلق بالمنهج فيستدعي البحث عن مواضع ذلك القصور في منهج تعليم اللغة العربية في المعاهد. يتحدد بهدي إطار الدر اسة في مشكلات منهج تعليم اللغة العربية من حيث الأهداف و المحتوى والطريقة و الوسائل التعليمية و التقويم و سوف تتخذ الدر اسة مكانها في المعاهد اللغوية التابعة لمؤسسة مسلمي أَسيا الخيرية في إندونيسيا. الكلمات الدالة: المشكلات المنهجية، انتقالية، الأداء اللغوي. 
فقد تبين للثُعوب والأمم في هذا العصر ما للغة العربية من أهمية في حياتها السياسية والاقتصادية والثقافية وغيرها، فتوضع المناهج وتفتح المدارس و المعاهد وتعد المعلمون لتعليمها. و اللغة العربية في إندونيسيا لها مكانة مرموقة في حياة شعبها. وكان دعاة العرب لهم الفضل و الثرف و التقدير في هذه المهنة، ثم تابعتها بعد ذلك الأجيال المتعاقبة من أبنائها

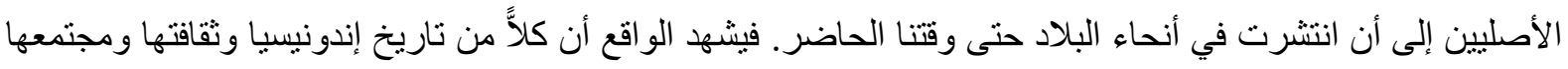

يزخر بصلات عميقة بالعرب ولغتهم وبالدين الإسلامي وتر اثثه، مما يؤدي إلى سهولة تعليم اللغة العربية لأبناء إندونيسيا. فمن أجل تحقيق فهم الإسلام في نفوس أبناء المسلمين أنشئت مراكز تعليم اللغة العربية في عهد مبكر من دخول الإسلام إلى الأرخبيل الإندونيسي (محمد الكافي، د ط: 47) ، بدءاً من الحلقات القر آنية في المساجد حتى إنشاء المدارس الإسلامية التي تستند إلى النظم التعليمية الحديثة و المطورة وانتشرت مر اكز تعليم اللغة العربية في مختلف مناطق هذه البلاد.

\section{اللغة العربية ووضها في إندونيسيا}

إن تاريخ تعليم اللغة العربية يبدأ منذ بزوغ شمس الإسلام في هذه البلاد باعتبار أن تعمها وتعليمها من صميم واجبات

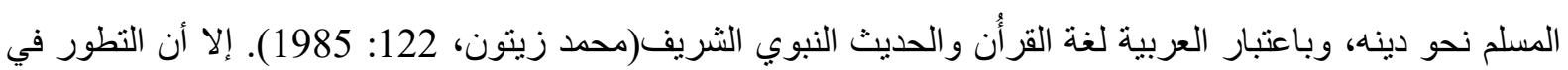
تعليم اللغة العربية يكون في بداية الألفينات، حيث تنشأ في إندونيسيا معاهد تعليم اللغة العربية والدراسات الإسلامية (مصطفى محمد نوري، 392: 2013). و أخص بالذكر هنا المعاهد التي تحت إشر اف مؤسسة مسلمي آسيا الخيرية، هذه المعاهد منتشرة في الجزر الكبيرة في إندونيسيا: في جزيرة جاوى، جزيرة سومطرة، جزيرة سلاويسي، جزيرة مالوكو و فى جزيرة كالمنتان.

و في السنوات الست الأخيرة ظهر تخصص تعليم اللغة العربية في بعض الجامعات الإسلامية الحكومية الإندونيسية على مستوى الماجستير والدكتوراه. هذا التخصص كان لا يتجاوز مرحلة البكالوريوس لعقود طويلة مما يقق وراء تدني المؤهل التعليمي لأغلبية معلمي اللغة العربية في إندونيسيا. إضافة إلى ذللك، بدأت تظهر بر امج تعليم اللغة العربية لأغر اض معينة مثل تعليم اللغة العربية لفهم القرآن الكريم، ظهر هذا في بعض المدن الإندونيسية ويتخذ مكانه في المساجد ومر اكز الإسلامية ويركز فيه الر اغبين في فهم القرآن الكريم ولكن

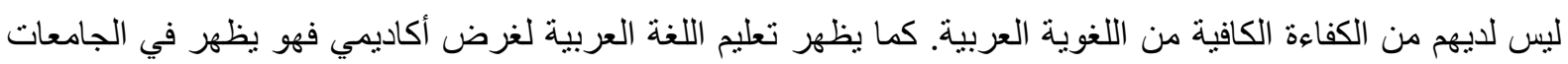

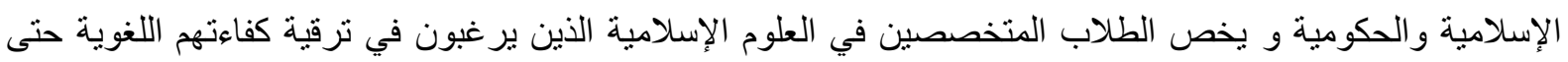

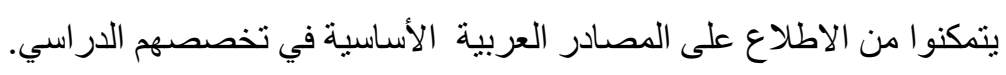
من علامة تطور مجال تعليم اللغة العربية في إندونيسيا أنه بدأ يستعين بالوسائل الإعلامية بصرية كانت أم سمعية بصرية، مثل تعليم اللغة العربية من خلال قنوات التلفزيون، من خلال المجلات، و عن طريق شبكة الإنترنت بل قد يتخذ بالوسائل الحديثة و هي الوسائل الرقمية.

إن استخدام هذه الوسائل الإعلامية تتكل تطورًا غير مسبوقًا في مجال تعليم اللغة العربية في إندونبسيا. وهذا يغير انطباع الجميع بأن هذا المجال تقليدي معزول ومنحصر في بيئات وحلقات دينية محددة ومن خلال وسائل تعليمية تقليدية، ومن ثَّم يرفع معنويات متعلمي اللغة العربية ومعلميها سيكولوجياً. 
تعليم اللغة العربية في إندونيسيا من حيث الأهداف وطرق التدريس

إن من العوامل الرئيسية المؤدية إلى انتشار اللغة العربية فى إندونبسيا هو العامل التربوي والذي يتمثل في برامج تعليم اللغة العربية التي تطورت تطورًا ملموسا من حين إلى حين. كما أن تعليم اللغة العربية قد مرت بمر احل مندرجة ــ سواء كانت من حيث الأهداف أو من حيث الطرق- (أحمد فؤاد أفندي، 409 : 4001 إس

يمكن ترنيبها كالآتي:

\begin{tabular}{|c|c|c|}
\hline الأهداف التعلمية و طرق التدريس & البر امج اللغوية & 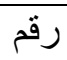 \\
\hline 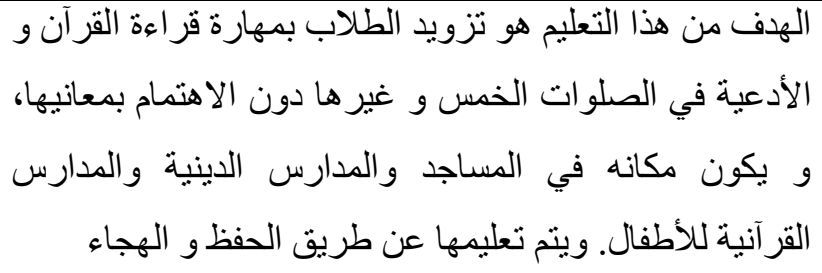 & تعليم اللغة العربية لفظياً & 1 \\
\hline 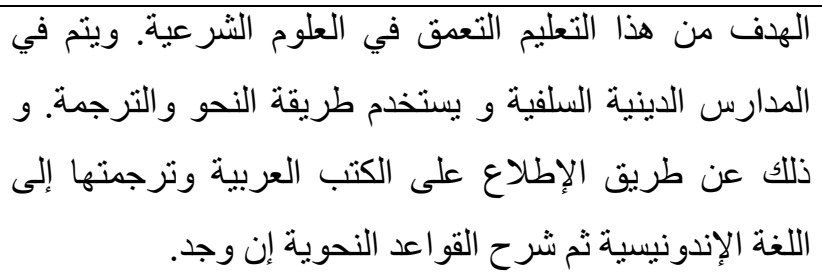 & تعليم اللغة العربية التقليدي. & 2 \\
\hline 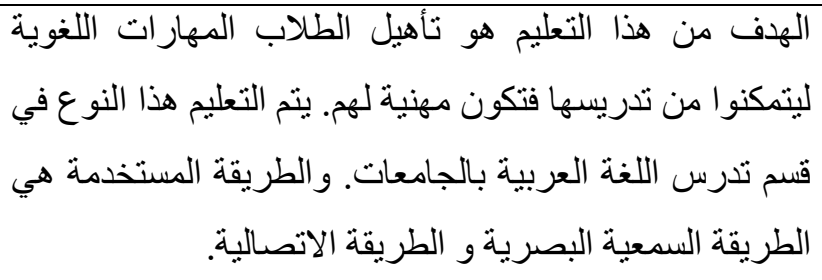 & قسم تدريس اللغة العربية & 3 \\
\hline التي يحتاجون إليها في من هذا تزويد المشال أعمالهم كالسياحة، بالمهارات والتجارة، اللغوية & دورات في اللغة العربية & 4 \\
\hline 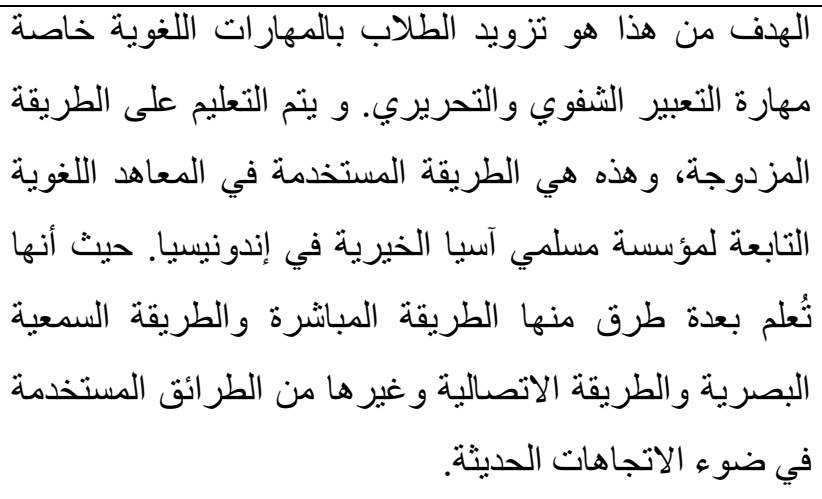 & التعليم في المعاهد اللغوية. & 5 \\
\hline
\end{tabular}


مما أثرنا إليه سايقا يتضح أن شأن تعليم اللغة العربية في إندونيسيا كان يمر بعدة مر احل، حيث بدأت من طريقة الحفظ و الترجمة لأغر اض دينية إلى أن يسير تعليمها على نمط منقدم، و ذلك في إطار تطور الاتجاهات الحديثة في تعليم اللغات الأجنبية. و للأهداف الأوسع و أشمل نطاقاً بالوسائل التكنولوجية الحديثة.

و الجدير بالذكر هنا أن هذه النطورات الملحوظة لا تضمن أن تعليم اللغة العربية في إندونيسيا أصبحت بعيدة من كل

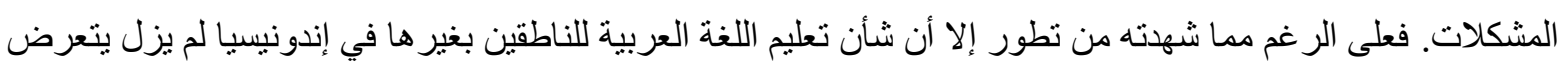
لمشكلات منهجية عديدة. و المعاهد اللغوية التابعة لمؤسسة مسلمي آسيا الخيرية لا تخلو من هذه المشكلات المنهجية.

\section{المشكلات المنهجية في تعليم اللغة العربية للناطقين بغيرها}

فيما ذكرنا سابقاً، أن تعليم اللغة العربية في إندونيسيا لم يزل يتعرض لبعض المشكلات المنهجية. يمكن ذكر أهمها فيما يلي:

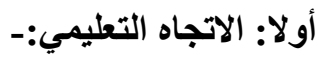

تتمثل هذه المشكلة في تعليم اللغة العربية الذي يسعى بصورة بالغة إلى تحقيق الأهداف الدينية ويتقيد باتجاه النحو والترجمة. يتركز التعليم في ضوء هذا الاتجاه على عملية تمكين الطلاب من فهم القرآن والأحاديث وغير هما من النصوص العربية الدينية (عبد الرحمن موسى أبكر ، 3: 1992).

إن تعليم اللغة العربية لتحقيق الأهداف الدينية ليس عيباً على الإطلاق وذلك لأنه يتو افق مع كون اللغة العربية كلغة دينية التي بها يفهم القرآن الكريم والأحاديث الثريفة، ويتو افق أيضا مع الحافز الداخلي للإندونيسيين لتعلم اللغة العربية. ولكن

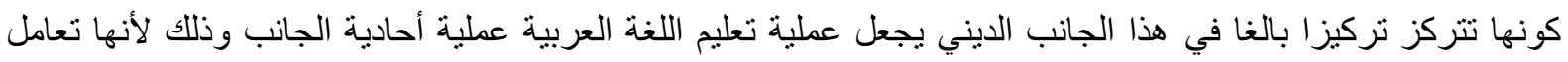

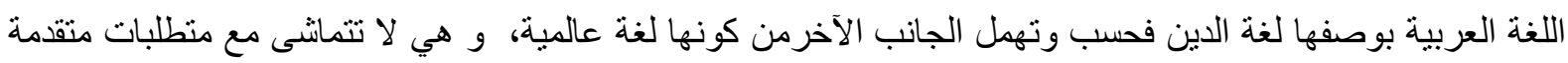
لتعليم اللغة العربية بصفتها لغة أجنبية (محمد زايد بركة، 33: 2000). وتعليم اللغة العربية في مثل هذا الاتجاه لا شك أنه يؤثر سلبياً على حصول الخبرات اللغوية المراد تزويدها للطلاب فالإهتمام البالغ بالقو اعد والترجمة على حساب المهارات اللغوية الأخرى التي لا تقل أهميتها من مهارة الاستماع والكلام

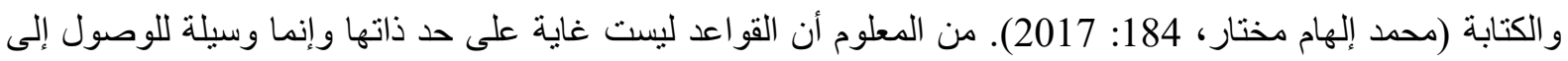
المهارات اللغوية و التي هي المقصودة في الاتجاهات الحديثة لتعليم اللغة العربية.

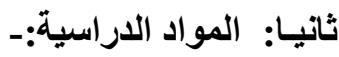
بإمكاننا تصنيف اتجاه تعليم اللغة العربية في إندونيسيا من حيث المواد الدراسية إلى اتجاهين: 1. تعلم اللغة العربية من خلال الكتب اللغوية والدينية، و يترتب على هذا الاهتمام الأكبر من الناحية الدينية في تعليم اللغة العربية. 2. تعلم اللغة العربية من خلال كتب السلسلة في تعليم اللغة العربية لغير الناطقين بها ، مثل: العربية للناشئين و العربية بين يديك.

اكتثف الباحث أن هذين الاتجاهين لم يقدما نتيجة مقبولة في تعليم اللغة العربية في إندونيسيا. فيعتبر الاتجاه الأول فانثل وذللك لأنه يهتم بالجانب المعرفي فحسب ويهمل الجانب الآخر الاتصالي. إضافة إلى ذلك فإن هذا الاتجاه يحتاج إلى المبدأ 
الأساسي في اللغة لأنها تبعد اللغة عن وظيفتها الاتصالية كما يفتقر إلى المبدأ المنهي لأنها تعتمد على الكتب الدينية ويترك كتب تعليم اللغة، وقد أشار إلى هذا أحمد شلبي في كتابه (1980:18)

أما الاتجاه الثاني فتكون المؤاخذات عليه لعدم مر اعاته و اهتمامه للمحتوى اللغوي الثقافي في اختيار المو اد الدراسية. مثال ذللك، كتاب "العربية للنانشئين" و كتاب "العربية بين يديك" إذا أردنا استخدامهما لتعليم اللغة العربية في إندو نيسيا فلا بد من

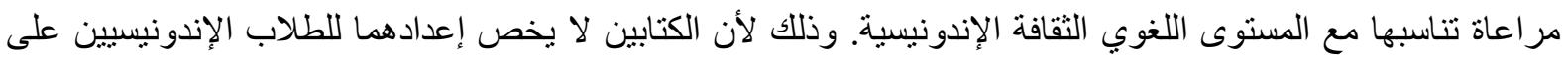
وجه الخصوص ولكن للناطقين بغير ها عامة.

ثالثا: طرائق التدريس:-

إن طريقة تدريس اللغة العربية الثائعة في في إندونيسيا هي طريقة النحو و الترجمة (نصر الدين،61: 2003) ذلك لأنها تسمح إلى حد كبير استخدام اللغة الأم في عملية التعليم والسبب يرجع إلى أن الكثير من مدرسي اللغة العربية في إندونيسيا لم يكن لديه الكفاءة من مهارة التحدث باللغة العربية.

ومن نقائص هذه الطريقة أنها تتطلب إلى أسس منهجية فضلا عن أسس لغوية تربوية. وقد أكد العلماء أنها ثبتت فثلها في تعليم اللغة العربية في معظم الدول الناطقة بغير العربية. و من نتائج التعليم على هذه الطريقة ــ على حد ما أثبتته الدراسات ـ أن الطلاب تخرجوا و هم ملمون بقو اعد اللغة العربية وفن الترجمة ولكن في الوقت نفسه مفتقرون إلى مهار ات الاتصـال

والتحدث باللغة العربية.

لا شك أن هناك محاو لات كثيرة في بعض الجامعات في استخدام طرق أخرى مثل الطريقة المباشرة والطريقة السمعية الثفوية، لكن هذه المحاو لات تبقى على حالها بسبب تدني مستوى المعلمين في الاتصال الثفوي باللغة العربية. رابعا: مشكلة الموارد البشرية:أما المشكلات التي تتعلق بمعلمي اللغة العربية في إندونيسيا فهي على النحو التالي: 1. إن معظم المعلمين لبس من تخصصهم تعليم اللغة العربية على وجه التحديد، إضافة إلى أن معلوماتهم حول تعليم

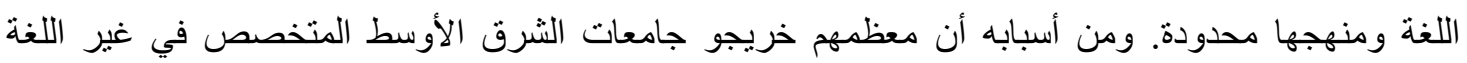

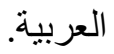
2. إن معظمهم لم يشتركو ا دورات تدريبية في إعداد المعلمين سو اء كان قبل الخدمة أو أثنائها مما يصعب عليهم من تحسين أدائهم التعليمي. 3. إن الأكثرية منهم لا يستطعون الاتصال باللغة العربية فيضطرون إلى استخدام اللغة الإندونيسية في عملية التعليم ، و هذا الأمر الذي يساعد شيوع استعمال طريقة النحو والترجمة في معاهد تعليم اللغة العربية في إندونيسيا. (فتحي

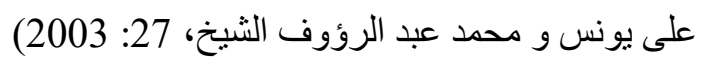
مما سبق، اكتشف أن القصور المذكورة في الموارد البشرية لا يأتي عفوًا و إنما تقف وراءها عدة عوامل أهمها فيما يلي: 1. عدم توفير قسم تعليم اللغة العربية خاصة على المستوى الماجستير والدكتور اه إلا في السنوات الأخيرة، فيصعب للمعلمين من تأهيل أنفسهم في هذا المجال، فيضطر بعضهم إلى اختيار التخصص الذي لا علاقة لها مهنيا ومعرفيا بكونهم معلمي اللغة العربية. 
2. قلة الدورات التدريبية التي يمكن أن ينمي بها قدرات المعلمين التعليمية.

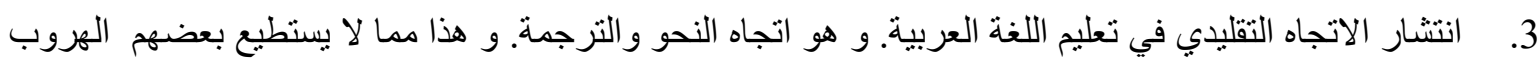

منه، فهم إنتاج هذا الاتجاه فلا يستغرب إذا تمكنوا من القو اعد والترجمة ولكن ضعفاء في المهار ات الاتصالية.

\section{تحليل وتقويم المنهج المتبع في المعاهد اللغوية التابعة لمؤسسة مسلمي آسيا الخيرية}

تعليم اللغة العربية للناطقين بغيرها في المعاهد اللغوية التابعة لمؤسسة مسلمي آسيا الخيرية يتم من خلال ما بسمى

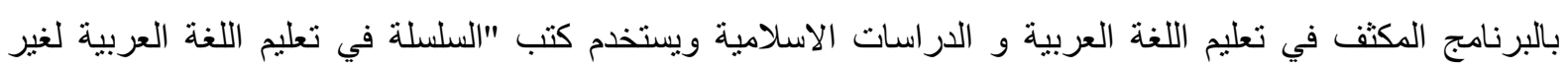
الناطقين بها" و كتاب "العربية بين يديك". و فيما يلي تحليل هذا المنهج اكتشافاً لما له من ايجابيات و ما عليه من سلبيات:

أو لاً: البنية

اثنملت هذا المنهج على العناصر الخمسة للمنهج وهي الأهداف و المحتوى وطرق التدريس و الوسائل التعليمية و التقويم مما يعني أنه منمانشي مع نظرية المنهج الحديثة التي تلزم توفر هذه العناصر الخمسة في المناهج المصممة. فالمنهج قابل للنطبيق حيث إن الكتاب المستخدم يعتمد على أسس تربوية ومنهجية سليمة بالإضافة إلى وجود إرشادات عامة للمعلم عند تقديم المو اد التعليمية.

وذلك لأن هذه المعاهد استمد منهجها من منهج قسم الإعداد اللغوي لمعهد العلوم الإسلامية والعربية التابعة لجامعة الإمام

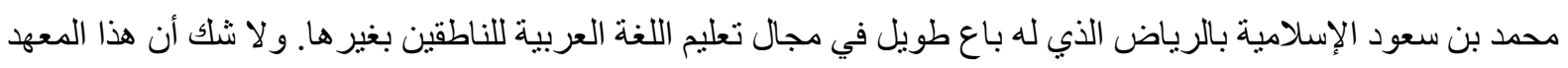

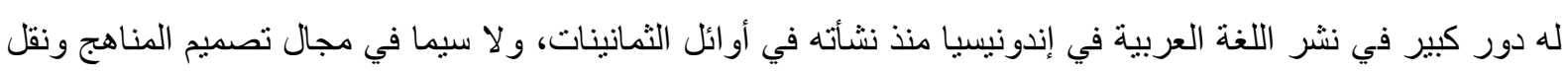
الخبرات التعليمية إلى المؤسسات الإسلامية التي لها اهتمام في تعليم اللغة العربية لأبناء هذا البلد. ثانياً: الأهداف

إن الأهداف المقررة في هذه المعاهد هو تخريج كوادر من الدعاة الملمين باللغة العربية و العلوم الإسلامية مما بساعدهم

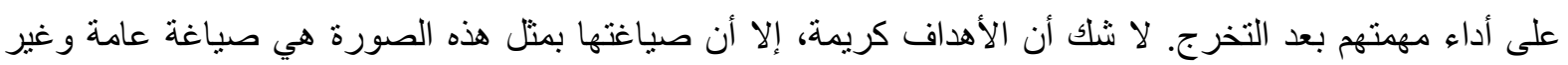

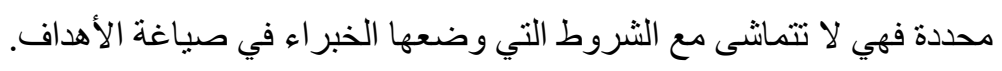

و من السبب الذي أدى إلى منل هذه الصياغة هو غياب المتخصصين في تعليم اللغة العربية للناطقين بغير ها فمعظم المدرسين تخرجوا من كليات الدر اسات الإسلامية وكلية الأدب وبالتالي لم يتأهلو التصميم المناهج.

ثالثاً: المحتوى

يستمد محتوى المنهج من كتب السلسلة في تعليم اللغة العربية التي أصدرتها جامعة الإمام محمد بن سعود الإسلامية

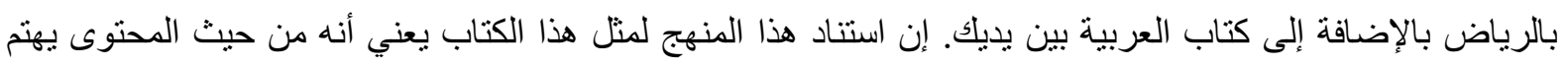
بتزويد الطالب بكل ما يحتاجون إليه من الكفاءات اللغوية العربية لغوية واتصالية وثقافية إذ أن كتب السلسلة المذكورة

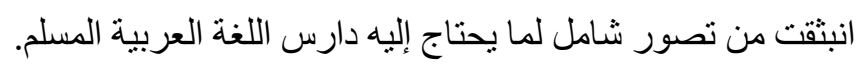

إن منطلق المنهج الذي ينبني على هذا التصور يعتبر شيئ محمود فإن أكثر من يقبل على تعلم اللغة العربية هم من

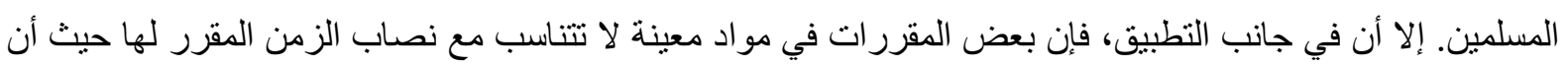


محتوى المادة كثيرة و الزمن المعد لها لايكفي لإنهاء المقرر في وقته فيضطر بعض المدرسين إلى عقد الدروس الإضافية حتى يتسنى لهم تقديم جميع محتوى المادة. رابعاً: الطريقة

يستخدم هذا المنهج الطريقة الانتقائية في عملية التعليم مما يعني أنه يعطي للمدرس حرية الاختيار لطرق التنريس الموجودة و المناسبة للمو اقف التعليمية التي يو اجهها المدرس فلا يتعصب بطريقة معينة من طرق تدريس اللغة بل يساعد المدرس على الاستفادة من محاسن طرق التدريس الموجودة و تجنب عيوبها في العملية التعليمية. إن استناد هذا المنهج بالطريقة الانتقائية يتو اكب مع المستجدات التربية الحديثة إذ إن التعصب لطريقة معينة يجعل التدريس غير مرنة وقد يؤدي إلى الملل في بعض الأحيان، إضافة إلى ذلك فإن لكل طريقة محاسنها و عيو بها. خامساً: الوسائل استعان هذا المنهج بشتى الوسائل التعليمية سمعية، وبصرية، وسمعي بصري. لاشك أن استخدام الوسائل التعليمية المختلفة يزيدها فعالية في عملية التدريس فإنها تثير اهتمام الدارسين إلى درجة كبيرة. ومن الوسائل التعليمية الموجودة في المعاهد اللغوية التابعة لمؤسسة مسلمي آسيا الخيرية، المعمل اللغوي.

ولكن الملاحظ في هذه المعاهد أن المعمل اللغوي لم يكن صالح الاستعمال، وذللك لأن استخدامه مقصوراً على مشاهدة الأفلام و الأخبار العربية. إضافة إلى ذلك فإن معظم المدرسين لم يجيدوا استخدام تلك الوسائل بصورة مثالية فإنهم في أغلب الأحيان لا يستخدمون الوسائل إلا السبورة و القلم و الكتاب المقرر فحسب. والسبب لأل الأن معظم المدريسن لم يمروا

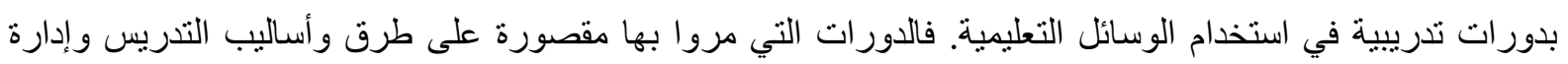
الصف.

سادساً: التقويم

نظام التقويم المتبع في هذا البرنامج يتمثل في الاختبار ات اللغوية التي يجلسها الدارسين في بداية الدراسة وفي أثنائها وفي

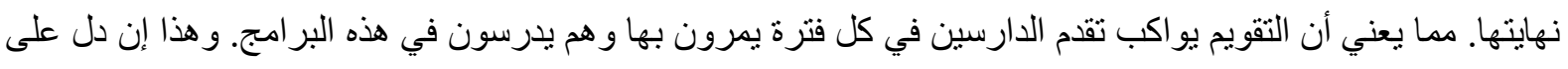

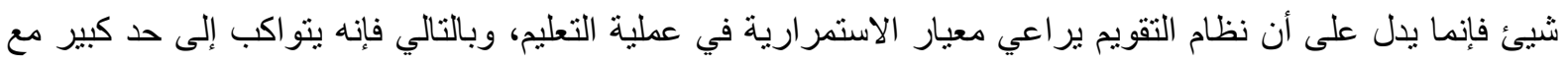

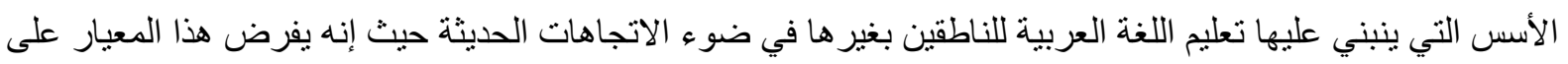
أن تسبق التقويم الععلية التعليمية وتلزمها وتتابعها (رشدي أحمد طعيمة، 283 : 1988) إلى أن نظام التقويم المتبع لقياس التحصيل اللغوي للمتعلم يتركز إلى حد كبير على اختبارات جو انب المعرفة اللغوية وقد لا يهنم بجانب الأداء اللغوي.

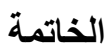

الخلاصة من المعلومات السابقة ذكرها، أن مناهج تعليم اللغة العربية في المعاهد اللغوية التابعة لمؤسسة مسلمي آسيا الخيرية في إندونيسيا بدأ يتجه إلى الاتجاهات الحديثة في مجال تعليم اللغة العربية للناطقين بغير ها، رغم أنها لا تزال تحتاج إلى مزيد من التطوير في جو انبها المختلفة، كالأهداف و المحتوى وطرق التدريس و الوسائل التعليمية و التقويم. 
فمن أجل تحسين وتطوير مناهج تعليم اللغة العربية للناطقين بغير ها ورفع مستو اها، يرجى من مسؤولي المؤسسة الإعتتاء بالتدريب المهني لمدرسي اللغة العربية في ضو ء الاتجاهات الحديثة في جميع جو انبه، والاهتمام بتوفير الوسائل التعليمية الحديثة مواكبة لمستجدات التربية الحديثة وعقد دورات تدربيبة لمشرفيها، إضافة إلى تزويد المكتبات بالكتب العربية الحديثة التي من شأنها تساعد الدارسين على زيادة ثروتهم اللغوية. 


\section{المصادر و المراجع}

نوري، مصطفى محمد وحفة إنتان. (2013). العربية الميسرة، جاكرنا، مكتب عارف للنشر طعيمة، رشدي أحمد. (1988). الأسس المعجية والثقاقية لتعليم اللغة العربية لغير الناطقين بغير ها، مكة المكرمة، وحدة

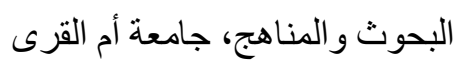

زينون، محمد. (1985). المسلمون في الشرق الأقصى، القاهرة, دار الوفاء

أفندي، أحمد فؤاد. (2001). خريطة تعليم اللغة العربي في إندونيسبا، مجلة اللغة والفنون، كلية الأداب بجامعة مالانج الحكو مية

شلبي، أحمد. (1980). تعليم اللغة العربية لغير العرب، مكتب النهضة المصرية، القاهرة جوهر، نصر الدين إدريس. (2003). الأفعال المتعدية بحروف الجر وتدريسها للإندونسيين، بحث تكميلي غير منشور مقدم لنيل درجة الماجستير، معهد الخرطوم للغة العربية

الكافي، محمد. (دون تاريخ طباعة). الإسلام في إندونسيسيا المعاصرة أبكر، عبد الرحمن موسى. (1992). و اقع تعليم اللغة العربية في الجامعات الإندونيسية الواقع و المستقبل، جاكرتا، معهد العلوم الإسلامية والعربية في إندونيسيا

بركة، محمد زايد. (2000) اللغة العربية لدى الناطقين بهاو الناطقين بغير ها، المجلة العربية للار اسات اللغوية، العدد 17، فبراير، معهد الخرطوم الدولي للغة العربية

يونس، فتحي على و محمد عبد الرؤوف الثيخ، (2003) المرجع في تعليم اللغة العربية للأجانب (من النظرية إلى

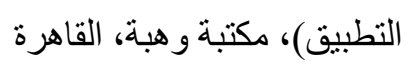

Muchtar, M. I. (2017). Contextual teaching and learning method in studying Arabic. Hunafa Jurnal Studia Islamika, Volume. 14 No.1, 175-188 , State Islamic Institut (IAIN), Palu. Central Sulawesi. 